\title{
ATUAÇÃO DO PROFISSIONAL DE FARMÁCIA PERANTE OS ERROS DE MEDICAÇÃO
}

\author{
ATTITUDES OF THE PHARMACY PROFESSIONALS REGARDING MEDICATION ERRORS \\ ACTUACÍON DEL PROFESIONAL DE LA FARMACIA ANTE DE LOS ERRORES DE LA \\ MEDICACIÓN
}

\author{
Ângelo Jesus (acj@estsp.ipp.pt)* \\ Mafalda Vasconcelos (mafalda_951@ hotmail.com)** \\ Agostinho Cruz (asc@estsp.ipp.pt)***
}

\begin{abstract}
RESUMO
O trabalho de equipa e as oportunidades para aprender com o erro são de máxima importância para a melhoria da segurança do doente, bem como para a promoção de uma cultura real e justa, na qual a notificação do erro e a sua resolução são encorajadas num ambiente livre de culpa mas responsável. Na maioria dos casos, o erro não é sinal de um mau profissionalismo, mas sim de um acompanhamento inevitável da condição humana, associado a sistemas altamente suscetíveis a erro. No presente artigo efetuamos uma revisão narrativa sobre erros de medicação, na perspetiva do Profissional de Farmácia, revisitando o tema e discutindo a evolução de conceitos e de taxonomias, assim como as principais causas desencadeadoras dos mesmos. Faz-se igualmente referência ao circuito do medicamento hospitalar e às opções desenvolvidas para a notificação do erro e outros incidentes.
\end{abstract}

Palavras Chave: erros de medicação, prescrição, dispensa, administração, notificação.

\begin{abstract}
Teamwork and opportunities to learn from errors are of utmost importance to improve patient safety and the promotion of a true and fair culture in which the reporting of errors and their resolution are encouraged in an responsible but free of guilt environment, leading to improvement of the Quality System. In most cases, error is not a sign of bad professionalism but rather an inevitable accompaniment of the human condition associated to systems that are highly susceptible to error. In this article the authorscarry out a narrative review of medication errors, from the perspective of the Pharmacy Professional, revisiting the topic and discussing the evolution of concepts and taxonomies as well as the primary precipitating causes. Reference is also made to the medication cycle in the hospital and the options developed for error notification.
\end{abstract}

Keywords: medication errors, prescription, dispensing, administration, notification.

\section{RESUMEN}

El trabajo en equipo y las oportunidades para aprender del error son de suma importancia para la mejoría de la seguridad del paciente, así como para promover una cultura real y justa, donde se anima a la notificación de la errata y a su resolución en un ambiente libre de culpa pero responsable. En la mayoría de los casos, el 
error no es ningún signo de mala profesionalidad, sino un acompañamiento inevitable de la condición humana, asociado a sistemas altamente susceptibles a equivocación. En este artículo efectuamos una revisión narrativa de los errores de medicación, desde la perspectiva del profesional de Farmacia, revisando el tema y discutiendo la evolución de conceptos y taxonomías, así como las principales causas desencadenantes. También hace referencia al circuito del medicamento hospitalario y a las opciones desarrolladas para la notificación del error y de otros incidentes.

Palabras clave: errores de medicación, prescripción, dispensación, administración, notificación.

* Instituto Politécnico do Porto, Escola Superior de Tecnologia da Saúde do Porto. Licenciado em Farmácia. Doutor em Ciências da Educação Coordenador de Curso de Licenciatura em Farmácia da ESTSP-IPP.

**Aluna Finalista da Licenciatura em Farmácia da Escola Superior de Tecnologia da Saúde do Porto

*** Instituto Politécnico do Porto, Escola Superior de Tecnologia da Saúde do Porto. Doutor em Ciências Biomédicas. Coordenador da Área Técnico Científica de Farmácia. Presidente da ESTSP-IPP

Submitted: 23rd December 2014

Accepted: 05th November 2015 


\section{INTRODUÇÃO}

A preocupação com a ocorrência de erros de medicação, intensificou-se a partir da publicação do relatório do Institute of Medicine intitulado To err is human: building a safer health system (Kohn, Corrigan, \& Donaldson, 2000). A expectativa que os profissionais de saúde seriam infalíveis, fez com que o erro fosse encarado como uma falta de cuidado, falta de atenção ou até mesmo falta de conhecimento; tornando-o deste modo, inaceitável (Diz \& Gomes, 2008). Esta perspectiva centrada no indivíduo, tende a resultar em actos inseguros, erros e infracções dos procedimentos por parte do profissional de saúde. Consequentemente, o erro é tratado pelo sistema através de medidas disciplinares, medo, culpa e humilhação (Anacleto, Perini, Rosa, \& César, 2005). Este tipo de abordagem enfatiza a punição como principal elemento de qualquer actividade correctiva. Ou seja, ao centrarmos apenas o profissional de saúde como origem de erro, corremos o risco de ignorar outros factores associados a um contexto sistémico. O ser humano falha, mas o erro deve ser confrontado como consequência e não como causa. Portanto, embora não seja possível alterar a condição humana, podem ser alteradas as condições em que o ser humano trabalha, através da criação de defesas no circuito do medicamento.

De acordo com Reason (2000), as defesas podem ser caracterizadas de acordo com a função, a que se propõem servir, e pelo modo como essas mesmas funções são executadas. As funções defensivas encontram-se geralmente operacionalizadas através de uma combinação de aplicações do tipo hard e soft. Defesas hard incluem dispositivos técnicos, sistemas de alarme e barreiras físicas. Enquanto as defesas soft correspondem por exemplo a legislação, regulamentação e a formação (Fragata e Martins, 2004).

Numa situação ideal, cada uma destas barreiras deveria estar intacta. Contudo, na realidade cada uma destas, encontra-se associada a uma imagem semelhante a uma fatia de queijo suíço em que, cada um dos seus vários buracos corresponde a uma determinada dificuldade ou falha (Cassiani et al., 2005). Segundo Fragata e Martins (2004), os erros resultam quase sempre não de actos isolados, mas de uma sucessão de falhas activas, passivas e faltas de defesa que criam entre si um jogo dinâmico, construindo assim "buracos" de segurança uma vez que, estes estão constantemente a alterar a posição entre si. Pontualmente, estes "buracos" alinham-se criando uma trajectória linear. Esse alinhamento momentâneo é conhecido como a "janela de oportunidade". Os erros de medicação constituem um exemplo disso mesmo (Cassiani et al., 2005).

Dados do Committee on Quality of Health Care in America (Kohn et al., 2000), mostram que os erros de medicação foram responsáveis por cerca de 7391 mortes anuais nos hospitais americanos e que, aproximadamente metade desses erros terão uma relação direta à falta de informação sobre a dose correta; os restantes estão relacionados com frequência e na via de administração. Segundo este relatório, cada doente admitido num hospital sofreria, por estimativa, de 1,4 erros de medicação durante a sua hospitalização e a cada 1000 prescrições executadas seriam encontrados 4,7 erros. Existe assim, uma estimativa de que 44.000 a 98.000 doentes morrem em cada ano, num total de 33.6 milhões de internamentos, devido a erros que poderiam ser evitados nos EUA, indicando que morrem mais americanos devido a este tipo de erro do que em acidentes de viação, SIDA e cancro da mama (Kohn et al., 2000). 
Os erros de medicação, para além de lesarem o doente, acarretam custos elevados para os sistemas de saúde (Cassiani et al., 2005) Estima-se que os custos anuais nos Estados Unidos resultantes da morbilidade e mortalidade relativos a terapêuticas medicamentosas inapropriadas sejam de 47 biliões de dólares, valor comparativamente superior ao gasto anualmente no tratamento da diabetes mellitus (Bates et al., 1998).

Ao melhor do nosso conhecimento, não existem estatísticas oficiais disponíveis sobre a realidade em Portugal embora este tema tenha sido já alvo de debate na imprensa (Agência Lusa de Notícias, 2008). Também os dados relativos a custos não foram alvo de divulgação. Todavia, tendo em consideração o que já foi debatido, será expectável que também em Portugal existam custos acrescidos para o Sistema Nacional de Saúde (SNS) devido a erros de medicação. Numa altura em que os cortes orçamentais assombram o SNS, é ainda mais relevante discutir defesas sistémicas aos erros de medicação.

Focando a nossa atenção nos Profissionais de Farmácia e no seu impacto no circuito do medicamento, no presente artigo pretende-se efectuar uma revisão narrativa da literatura que suporta a actuação destes profissionais perante os erros de medicação. É fornecido um enquadramento terminológico, promove-se uma reflexão sobre as causas dos erros de medicação e formas de prevenção, analisa-se o circuito do medicamento hospitalar e culmina-se nas estratégias para notificação de erros de medicação.

\section{MÉTODOS}

O presente artigo apresenta-se na forma de uma revisão narrativa, no entanto foram tomadas providências de forma a sistematizar as estratégias de busca e seleção de informação. Efetuou-se uma pesquisa online nas bases de dados Pubmed/MEDLINE; ISI WEB OF KNOWLEDGE, Scientific Electronic Library Online e Red de Revistas Científicas de América Latina y el Caribe, España y Portugal, usando as seguintes palavras-chave: medication error; error notification, dispensing; distribution; administration; monitoring

Foram analisados títulos e/ou abstracts de artigos, com full text disponível, limitados ao período de Janeiro de 1990 a Dezembro de 2013 e aos idiomas inglês, português, espanhol e italiano de acordo com a relevância para o tópico em questão. Foram também incluídas referências citadas nos artigos da pesquisa inicial.

\section{CONCEITOS, TERMINOLOGIAS E TAXONOMIAS}

Um dos obstáculos encontrados para o estudo e prevenção de erros na medicação está associado a uma falta de padronização e a uma multiplicidade da terminologia utilizada para os classificar (Ferner \& Aronson, 2006; Aronson, 2009). De facto, o interesse pelo estudo da dinâmica do erro fez nascer toda uma terminologia que é necessário utilizar com precisão.

Retomamos aqui a definição de erro de medicação, conforme definido pela National Coordinating Council for Medication Error Reporting and Prevention (NCCMERP)(1998) "any preventable 
event that may cause or lead to inappropriate medication use or patient harm while the medication is in the control of the health care professional, patient, or consumer. Such events may be related to professional practice, health care products, procedures, and systems, including prescribing; order communication; product labeling, packaging, and nomenclature; compounding; dispensing; distribution; administration; education; monitoring; and use."

Atualmente, três organizações mundiais têm vindo a promover o alcance de uma taxonomia consensual: a National Coordinating Council for Medication Error Reporting and Prevention (NCCMERP), a American Society of Hospital Pharmacists (ASHP) e a Organização Mundial de Saúde (OMS)(Anacleto, Rosa, Neiva, \& Martins, 2010).

No documento de 1998, a NCCMERP publicou uma taxonomia referente a erros de medicação, classificando estes em diversos tipos e subtipos. Mais tarde em 2001, essa mesma instituição publicou uma atualização dessa classificação, identificando nove categorias distintas de erros em função da gravidade, considerando se existiu ou não danos ao doente, qual a duração e a extensão deste dano e se foi necessário algum tipo de intervenção.

Posteriormente em 2003, um grupo de farmacêuticos hospitalares espanhóis- Grupo Ruiz-Jarabo, com a permissão da United States Pharmacopeia - USP e sob coordenação do Institute for Safe Medication Practices - ISMP de Espanha, elaborou uma adaptação dessa classificação (Otero, Jané, Alonso, \& Encinas, 2003). Em 2008, também essa proposta sofreu uma actualização (Otero et al., 2008).

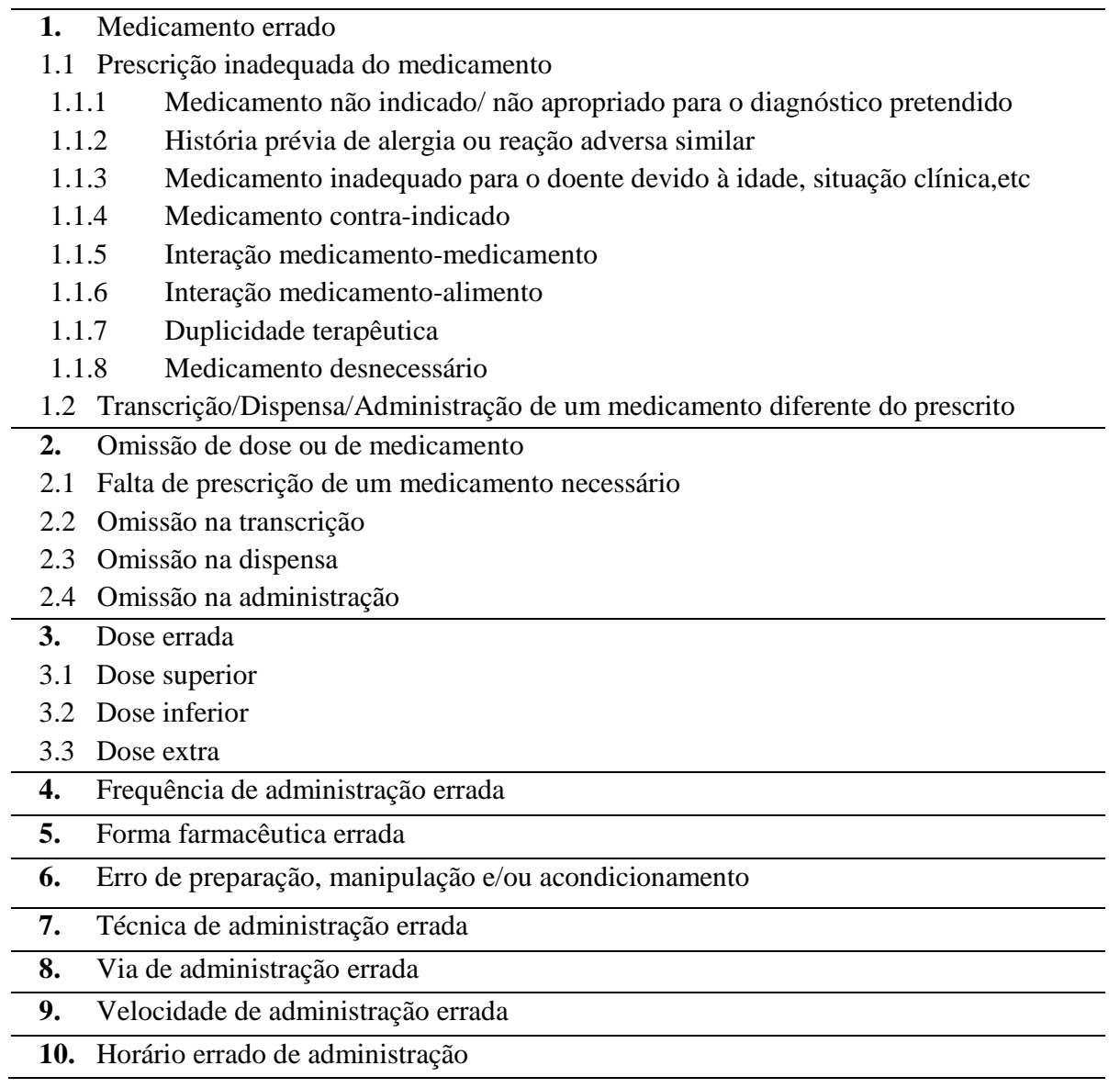




\begin{tabular}{l}
\hline 11. Doente errado \\
\hline 12. Duração de tratamento errada \\
12.1 Duração superior \\
12.2 Duração inferior \\
\hline 13. Monitorização insuficiente do tratamento \\
13.1 Falta de revisão clínica \\
13.2 Falta de controlo analítico \\
\hline 14. Medicamento em estado de deteorização \\
\hline 15. Falta de adesão do doente \\
\hline 16. Outros tipos \\
\hline 17. Não se aplica \\
\hline Tabela 1 - Classificação dos Erros de Medicação de acordo com o Grupo Ruiz-Jarabo \\
\hline
\end{tabular}

Esta atualização foi motivada por: a) outras classificações utilizadas por sistemas de notificação de incidentes ocorridos após a realização da primeira versão; b) sugestões feitas por profissionais de saúde na primeira versão; c) experiência gerada a partir da análise de erros ocorridos em hospitais espanhóis e notificados ao ISMP Espanha (Otero et al., 2008). O Grupo Ruiz-Jarabo diferencia uma classificação dos tipos de erros de medicamentos (Tabela 1) e estabelece uma categorização da gravidade dos mesmos (Tabela 2). Devemos realçar que esta classificação não cria categorias eliminatórias, ou seja, um erro de medicação (ocorrência) poderá ser incluída em mais do que um tipo ou subtipo.

\begin{tabular}{|c|c|}
\hline Categoria & Definição \\
\hline $\mathbf{A}$ & $\begin{array}{l}\text { Circunstâncias ou situações capazes de produzir um erro, mas que são } \\
\text { detetadas e resolvidas antes de afetar o doente }\end{array}$ \\
\hline $\mathbf{B}$ & $\begin{array}{l}\text { O erro afeta o doente mas não causa nenhum dano, não sendo necessária } \\
\text { monitorização ou intervenção }\end{array}$ \\
\hline $\mathbf{C}$ & $\begin{array}{l}\text { O erro afeta o doente e causa dano, sendo uma intervenção e } \\
\text { monitorização necessárias para confirmar a causa do dano }\end{array}$ \\
\hline $\mathbf{D}$ & O erro causa dano que é impossível de ser determinado \\
\hline $\mathbf{E}$ & $\begin{array}{l}\text { O erro contribui para danos temporários no doente (reversível) e requere } \\
\text { intervenção }\end{array}$ \\
\hline $\mathbf{F}$ & $\begin{array}{l}\text { O erro contribui para danos temporários (reversíveis) e requere uma } \\
\text { admissão hospitalar prolongada }\end{array}$ \\
\hline $\mathbf{G}$ & O erro contribui para danos permanentes no doente (irreversíveis) \\
\hline $\mathbf{H}$ & $\begin{array}{l}\text { O erro coloca em risco de vida o doente e a intervenção é necessária para } \\
\text { assegurar a sua sobrevivência }\end{array}$ \\
\hline I & O erro contribui para a morte do doente \\
\hline
\end{tabular}

Note-se que a definição de erro de medicação difere de "reação adversa". De acordo com a definição da Organização Mundial de Saúde (OMS) uma reação adversa a medicamentos (RAM) é uma resposta prejudicial e indesejada a um medicamento, que ocorre com doses habitualmente usadas para profilaxia diagnóstico ou tratamento, e em que existe um nexo de causalidade entre a ocorrência adversa e o medicamento. Esta definição exclui falhas terapêuticas, envenenamentos acidentais ou intencionais (ex: overdose) e drogas de abuso. A definição de RAM foi, entretanto, alterada pela Diretiva 2010/84/EU do Parlamento Europeu e do Conselho de 15 de dezembro pelo que a partir de julho de 2012 se define como 'uma reação nociva e não intencional a um 
medicamento (Herdeiro, Ferreira, Ribeiro-Vaz, Polónia, \& Costa-Pereira, 2012). A reação adversa a medicamento é considerada como um evento inevitável, ainda que se conheça a sua possibilidade de ocorrência, enquanto os erros de medicação, por definição, podem ser prevenidos.

\section{CAUSAS DE ERROS DE MEDICAÇÃO}

A análise dos erros ocorridos nos Estados Unidos da América e reportados a instituições como a FDA (MEDWatch Program) e USP-ISMP (Medication Errors Reporting Errors), mostra que as causas dos erros são multifatoriais. Entre as principais causas estão: falta de conhecimento sobre os medicamentos, falta de informação sobre os doentes, violação de regras, deslizes e lapsos de memória, erros de transcrição, falhas na interação entre os serviços, falhas na conferência da medicação, problemas relacionados com dispositivos de infusão de medicamentos, monitorização inadequada do doente, problemas no armazenamento e dispensa, erros de preparação e falta de padronização dos medicamentos. A literatura é díspar, no que diz respeito à categorização de causas de erros de medicação (National Coordinating Council for Medication Error Reporting and \& Prevention, 1998; Kohn et al., 2000; Otero et al., 2003; Cohen, 2006; Otero et al., 2008; Anacleto et al., 2010). No entanto todas as contribuições até ao momento podem ser sistematizadas nos dez fatores descritos abaixo. O Profissional de Farmácia deve estar sensibilizado para cada um destes fatores, de forma a prevenir a ocorrência de erros de medicação.

\subsection{INFORMAÇÃO RELATIVA AO DOENTE}

De modo a orientar a terapêutica adequada ao doente, os profissionais de saúde devem prontamente assegurar a recolha de informações clínicas relacionadas, nomeadamente a idade, o peso, o histórico de alergia a alimentos ou fármacos, gravidez, e toma concomitante de outros fármacos. No âmbito hospitalar, deverão constar na ficha do doente os dados de monitorização, tais como: informações detalhadas relativas a exames laboratoriais; deteção de sinais vitais; listagem de medicamentos utilizados e respetiva evolução de doença.

\subsection{INFORMAÇÃO RELATIVA AO MEDICAMENTO}

Para minimizar o risco de erros, os profissionais de saúde devem ter um acesso rápido a informação atualizada acerca dos medicamentos. Este acesso pode ser efetuado através de textos de referência, protocolos, sistemas e bases informáticas. Atualmente existem diversos recursos online, que podem auxiliar os Profissionais de Farmácia, nomeadamente o Prontuário Terapêutico (www.infarmed.pt/prontuário/index.php), Formulário Nacional Hospitalar do Medicamento (www.infarmed.pt/formulario), a base de dados Infomed (/www.infarmed.pt/infomed/inicio.php) ou mesmo a Agência Europeia do Medicamento (www.ema.europa.eu/).

\subsection{COMUNICAÇÃO RELATIVA AO MEDICAMENTO}

A comunicação é fundamental em todos os sectores de atividade mas assume especial importância no sector da saúde (Aguiar, 2004). As organizações de saúde devem trabalhar no sentido de promover a redução de barreiras de comunicação entre os profissionais de saúde (p.e. padronização de formas de prescrição e demais informações acerca dos medicamentos de modo a 
evitar erros de interpretação). Isto implica uma formação assertiva para a comunicação em Farmácia, por parte dos profissionais.

\subsection{ROTULAGEM, EMBALAGEM E DESIGNAÇÃO DO MEDICAMENTO}

De forma a facilitar uma adequada identificação e uso de medicamentos, os laboratórios produtores, instituições reguladoras, organizações de saúde e especialmente os serviços farmacêuticos/ farmácias deveriam assegurar que todos os medicamentos possuem rótulos claros e identificações diferenciadas para medicamentos com nome e pronúncia semelhantes. Contudo, no crescente mercado de genéricos e a enorme variabilidade de apresentações comerciais, a maioria dos laboratórios procuram criar um design para as embalagens e rótulos das suas especialidades de modo a alimentar uma determinada imagem de marca característica. Dado o número cada vez maior de especialidades e apresentações farmacêuticas que são comercializadas por alguns laboratórios, ocorre a possibilidade destas características coincidirem em certos aspectos, tais como o tamanho e cores da embalagem, culminando no aparecimento de especialidades que têm uma aparência muito similar (López, Merino, \& Alvaréz, 2002; López \& Muñoz, 2002).

Esta semelhança ao nível do design das embalagens (look-alike), pode ser promotor de erros de medicação, em 5 situações distintas (Jesus \& Ferreira, 2012) quando a cartonagem é semelhante e:
a) substância activa distinta;
b) número de unidades distinto;
c)via de administração distinta;
d) dosagem distinta;
e) forma farmacêutica distinta.

Existem ainda situações onde as semelhanças ortográficas e fonéticas nas designações dos medicamentos, podem ser causadoras de erro (Jesus \& Ferreira, 2012). Este tipo de situações volta a reforçar a importância do profissional de saúde, devidamente habilitado e atento aquando da dispensa de medicação. Sobre este tópico recomendamos a leitura da Norma 020/2014 da Direção Geral de Saúde - Medicamentos com nome ortográfico, fonético ou aspeto semelhantes.

\subsection{ARMAZENAMENTO E DISPENSA DE MEDICAMENTOS}

Muitos dos erros de medicação podem ser prevenidos através da implementação de uma estratégia de redução da disponibilidade de medicamentos ao nível dos diferentes setores (p.e. enfermarias), restringindo deste modo o acesso a medicamentos potencialmente perigosos e fazendo recurso a sistemas de dispensa de medicamentos que disponibilizem o medicamento somente no momento de utilização, diminuindo assim a ocorrência de erros (Campanella, Fraguglia, Ferelli, Rossi, \& Cericola, 2013). Uma das opções viáveis para diminuir o número de erros de medicação e conseguir igualmente uma melhor gestão do medicamento, passa pela implementação de Sistemas de Distribuição por Reposição de Stocks Nivelados, como por exemplo o sistema Pyxis®. O sistema semi-automático Pyxis ${ }^{\circledR}$ é um sistema computorizado composto por um conjunto de armários controlados eletronicamente, geridos por um software que se encontra em comunicação com as aplicações informáticas existentes, permitindo a oportunidade de este ser controlado por 
profissionais de saúde pertencentes aos serviços farmacêuticos e enfermaria (Miranda et al., 2012). Este sistema possibilita a determinação dos valores de stock mínimo e máximo para cada medicamento, tendo por base os registos de consumo médio. Sempre que os stock mínimo é atingido, é gerado um alerta nos serviços farmacêuticos, que são depois responsáveis pela reposição. $\mathrm{O}$ acesso aos medicamentos fica sempre sujeito a uma identificação do profissional de saúde e depende igualmente da prescrição eletrónica efetuada para cada doente.

Também a distribuição individual diária em dose unitária provou ser um sistema de distribuição eficaz e com resultados satisfatórios na redução de erros de medicação. Sobre este tópico aconselhamos a leitura das contribuições de Couto (2013).

\subsection{DISPOSITIVOS MÉDICOS ASSOCIADOS À ADMINISTRAÇÃO DE MEDICAMENTOS}

O design de alguns dispositivos utilizados no momento de administração de medicamentos pode facilitar a ocorrência de erros na medicação (p.e. bombas de infusão com fluxo livre para a administração de medicamentos intravenosos, conexões de tubos e cateteres compatíveis para a administração de medicamentos intravenosos e dietas. Compete aos Profissionais de farmácia, o desenvolvimento de ferramentas que permitam minimizar a ocorrência de erros de medicação associados à utilização indevida de dispositivos médicos, e deste modo facilitar o trabalho das equipas clínicas e assegurar uma terapêutica eficaz (Ferreira, Correia, \& Santos, 2012).

\subsection{ATUALIZAÇÃO DOS PROFISSIONAIS}

Os sistemas e serviços de saúde por todo o mundo estão em constante evolução, colocando, em análise crítica a formação e o treino requerido aos profissionais de saúde. Torna-se relevante perceber o que aprendem e acima de tudo, como aprendem (Jesus, 2014). Todos os pontos acima discutidos, apontam para a necessidade da existência de Profissionais de Farmácia devidamente habilitados, que sejam capazes de reconhecer a terapêutica farmacológica apropriada assim como estarem atualizados face ao corrente estado de conhecimento através de literatura científica, consultando os seus colegas e outros profissionais de saúde e participando num sistema de programas de formação contínua. Será ainda pertinente que os Profissionais de Farmácia possam participar no processo de monitorização terapêutica (incluindo a avaliação da terapêutica apropriada, administração medicamentosa ajustada e deteção de possíveis duplicações da terapêutica; rever a ocorrência de possíveis interações; e executar avaliações de dados clínicos e laboratoriais). O seu envolvimento em atividades de avaliação auxilia o alcance de um eficaz, seguro e racional uso de medicamentos. Portugal continua a ser um dos poucos países Europeus que aposta na formação superior dos seus Profissionais de Farmácia, através da Licenciatura ou do Mestrado Integrado, constituindo assim uma força de trabalho altamente qualificada (Vasconcelos \& Jesus, 2014).

Finalmente, Profissionais de Farmácia devem ser conhecedores e estarem adaptados quer aos sistemas de informação associados (prescrição da medicação, controlos por código de barras; sistemas semi-automatizados de distribuição), quer às políticas de distribuição e procedimentos pré-estabelecidos pela organização em causa, de modo a promover uma segura distribuição de 
medicamentos aos doentes. É também pertinente que os próprios profissionais sejam incluídos nas equipas que formulam os procedimentos internos dos serviços farmacêuticos/farmácia.

\subsection{EDUCAÇÃO DO DOENTE}

O doente possui um papel preponderante e vital na prevenção de erros de medicação ao receber informação pertinente acerca dos medicamentos e ao ser encorajado a perguntar e obter respostas satisfatórias relacionadas ao medicamento. Doentes que conhecem as designações e as doses dos seus medicamentos, as razões do uso de cada um dele e a forma de administração, encontram-se numa excelente posição para auxiliar e reduzir a probabilidade de ocorrência de erros.

Deste modo, os profissionais de saúde devem não só informar os doentes a protegerem-se de eventuais erros de medicação, como também alcançar neles uma promoção de melhoria da qualidade dos serviços.

\subsection{GESTÃO DE RISCO E PROCESSO DE QUALIDADE DOS SERVIÇOS}

As organizações de saúde devem possuir sistemas que permitam identificar, documentar, analisar e reduzir o risco da ocorrência de erros de medicação. A cultura de segurança não punitiva deve ser assim cultivada de modo a encorajar a sincera divulgação e oportunidades de erros no sistema. Estratégias de controlo tornam-se estritamente necessárias como meio de garantir a qualidade dos sistemas de utilização de medicamentos.

\subsection{FATORES FISIOLÓGICOS, AMBIENTAIS E PSICOLÓGICOS}

Na génese do erro encontram-se também presentes fatores fisiológicos, ambientais e psicológicos. Todos estes condicionam a performance humana, interferindo no desempenho de tarefas executadas e contribuindo deste modo para evitar ou favorecer a ocorrência do erro (Bulhões, 2001)

Tem sido discutido o impacto negativo provocado pela fadiga e a privação de sono, devido essencialmente à sobrecarga horária praticada pelos profissionais no sector da saúde, sobre a capacidade de resposta física e psíquica dos indivíduos. A título de exemplo, Dawson e Reid (1997) explicam que a performance psicomotora de um profissional de saúde após um turno de vinte e quatro horas é semelhante à de um indivíduo legalmente embriagado. Também a Agency for Healthcare Research and Quality (2003) relata que os erros relacionados com técnicas e procedimentos surgem com maior prevalência durante o turno da noite e quando ocorre uma redução do período de sono por parte dos profissionais.

As questões de foro pessoal e o stress podem também acarretar um impacto negativo na performance do individuo, culminando numa perda da sua capacidade de atenção. O stress fundase no resultado de interações entre as solicitações externas e a capacidade de resposta do indivíduo.

O fator "tempo" também não deve ser desvalorizado (Diz \& Gomes, 2008). Segundo Barros e colaboradores (2006) existe quase $100 \%$ de probabilidade de um profissional errar quando disponibiliza para um diagnóstico um minuto do seu tempo. Essa probabilidade é de cerca de $90 \%$ para 5 minutos, $10 \%$ em vinte minutos e $1 \%$ após um período de meia hora. Ainda em relação aos 
fatores desencadeantes de erros na medicação, é fulcral ter em atenção as causas residentes e inerentes a falhas latentes do sistema como ausência de protocolos e deficiente manutenção de aparelhos (Fragata \& Martins, 2004).

\section{CIRCUITO DO MEDICAMENTO HOSPITALAR}

O circuito do medicamento hospitalar, constitui um processo multidisciplinar que envolve três áreas: medicina, farmácia e enfermagem. Inicia-se no momento da prescrição médica, passa pela verificação, distribuição e dispensa executada pelo Profissional de Farmácia, terminando com a transcrição da prescrição e administração propriamente dita pelo enfermeiro. Portanto, encontramse envolvidos vários profissionais, e todos eles devem cooperar na excelência dos cuidados de saúde prestados, tendo em consideração que cada um possui competências e conhecimentos próprios (Nadzam \& Loeb, 1998; Cassiani et al., 2005; Crujeira et al., 2007; Coimbra, 2008; Diz \& Gomes, 2008).

Em 1989, a Joint Commission on Accreditation of Healthcare Organizations (JCAHO) identificou cinco processos associados ao circuito do medicamento hospitalar: seleção e aquisição do medicamento; prescrição; preparação e dispensa; transcrição e administração; e monitorização do doente acerca dos efeitos do medicamento (Nadzam \& Loeb, 1998). Vários estudos têm desde então procurado quantificar os erros de medicação que ocorrem em cada fase. Em 1995, Leape e colaboradores, relataram que 39\% dos erros de medicação ocorrem durante o processo de prescrição dos medicamentos, $12 \%$ na transcrição, $11 \%$ na dispensa e $38 \%$ na administração. Já em 2003, Runciman e colaboradores analisam o contexto australiano e identificam $2,4 \%$ de erros ao nível da prescrição. Ao nível da dispensa os valores são bastante reduzidos $(0,4$ a $0,8 \%)$, no entanto encontram uma disparidade interessante ao nível dos erros de medicação aquando da administração, afirmando que $15 \%$ a $20 \%$ decorrem quando é utilizado o stock da enfermaria, enquanto nas situações onde existe distribuição individualizada por doente, os erros assumiam valores de 5\% a 8\%. Seis anos depois, em 2009, um estudo levado a cabo por Benkirane e colaboradores, identificou que $71,1 \%$ dos erros de medicação acontecem aquando da prescrição, $21,2 \%$ aquando da administração, $5,7 \%$ aquando da transcrição e $2 \%$ aquando da dispensa. Um estudo sobre erros de medicação em unidades pediátricas e de cuidados intensivos (Wang et al., 2007), demonstrou que 54\% dos erros estavam associados à prescrição, $32 \%$ à transcrição da ordem médica, $12 \%$ ao nível da administração e finalmente $1,3 \%$ e $0,2 \%$ estavam associados à monitorização e dispensa respetivamente. Note-se que cada um destes exemplos se referem a contextos específicos e as suas conclusões não devem ser generalizadas. Contudo devem alertarnos para o real perigo dos erros de medicação e a necessidade de criar estratégias de defesa e minimização do risco.

Alguns destes princípios, são avançados por Womer e colaboradores (2002) e resultaram em melhorias para o circuito do medicamento, promovendo a redução de erros que atingem o doente tais como: eliminar as punições; aprender com o erro; simplificar o circuito do medicamento; padronizar os processos; utilizar procedimentos que estimulem os indivíduos a seguirem o caminho certo e reduzir a transferência de atividades entre profissionais e/ou grupos de pessoas. 


\section{A NOTIFICAÇÃo DE ERROS DE MEDICAÇÃo E OUTROS INCIDENTES}

Tobaiqy e Stewart (2013) procuraram as principais barreiras inerentes ao processo de notificação de erros nos sistemas de saúde. Os entrevistados apontaram como três principais barreiras: a falta de consciência do individuo, a sobrecarga no trabalho e limitação de tempo para expor a situação e a indisponibilidade de formulários de reportação de erros ao nível do sistema. As sugestões preferenciais que os autores apontaram para a melhoria da deteção, reportação e redução de erros de medicação, incluíram: ações de formação contínuas por parte dos profissionais de saúde (p.e. palestras, simpósios e conferências) (51,8\%); implementação de um sistema de reportação de erros online $(39,3 \%)$; formação focada em guidelines de prevenção de erros $(29,3 \%)$; anonimato na divulgação (25,0\%); adoçam de uma cultura não punitiva por parte do sistema $(21,4 \%)$ e apoios financeiros ligados ao preenchimento dos respetivos formulários (14,3\%).

A notificação de erros de medicação, ou outros incidentes de segurança foi alvo de uma recomendação da União Europeia - Recommendation Rec (2006)7 of the Committee of Ministers to member states on management of patient safety and prevention of adverse events in health care. Nesta recomendação está prevista a criação e desenvolvimento de um sistema de notificação em caso de incidentes de segurança, com o objetivo de melhorar a segurança do doente; este sistema deve ser independente dos restantes processos de regulação, não punitivo, anónimo, voluntário e ser concebido de forma a incentivar os prestadores de cuidados de saúde e cidadãos, a notificar incidentes médicos a incidentes de segurança. Em Portugal, o Sistema implementado denomina-se NOTIFICA - Sistema Nacional de Notificação de Incidentes (anteriormente denominado Sistema Nacional de Notificação de Incidentes e Eventos Adversos) e encontra-se disponível online através do endereço https://www.dgs.pt/formulario-notifica.aspx .

O Profissional de Farmácia, enquanto parte integrante da equipa multidisciplinar de saúde, deve estar sensibilizado para a existência e para a operacionalidade deste sistema de notificação, contribuindo para uma cultura de segurança do doente.

\section{CONSIDERAÇÕES FINAIS}

Os erros de medicação são uma condição inerente ao processo de prescrição, dispensa e administração de medicamentos. Mais do que focar a culpabilização, importa definir estratégias de defesa, de minimização e de notificação dos erros de medicação. O Profissional de Farmácia, enquanto membro integrante de uma equipa multidisciplinar deve estar consciente das potenciais causas dos erros de medicação e intervir aos vários níveis de gestão e execução para o desenvolvimento de protocolos e procedimentos que permitam uma diminuição significativa das oportunidades de erro. Mais ainda, na ocorrência do erro deverá ser executada uma investigação pronta e intensiva acompanhada por um processo de análise multidisciplinar do sistema em causa de modo a desvendar as causas próximas e sistémicas dos erros. A notificação do erro de medicação deve ser igualmente divulgada e estimulada junto dos profissionais, para que se possa contribuir para uma melhoria contínua e significativa da segurança dos doentes. 


\section{REFERÊNCIAS}

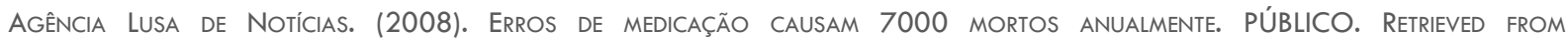
HTTP://WWW.PUBLICO.PT/SOCIEDADE/NOTICIA/ERROS-DE-MEDICACAO-CAUSAM-7000-MORTOS-ANUALMENTE- 1347542

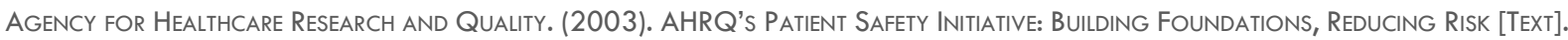
RETRIEVED AUGUST 19, 201 4, FROM HTTP://WWW.AHRQ.GOV/RESEARCH/FINDINGS/FINAL-REPORTS/PSCONGRPT/INDEX.HTML

Aguiar, A. (2004). A farmáCIA E A COMUnicaÇÃo (1 A Ed.). AJE.

Anacleto, T. A., Perini, E., Rosa, M. B., \& César, C. C. (2005). Medication errors and drug-dispensing systems in a hospital PHARMACY. CLINICS, 60(4), 325-332.

Anacleto, T. A., Rosa, M. B., Neiva, H., \& Martins, M. A. (2010). Erros de Medicação. Pharmacia Brasileira.

ARONSON, J. K. (2009). Medication errors: Definitions ANd ClassifiCATION. British Journal Of Clinical Pharmacology, 67(6), 599604.

Barros, P. P., Fragata, J., Santos, S., Ferreira, S., Bilbao, M., Fragata, I., \& França, M. (2006). Risco Clínico - Complexidade e Performance. Almedina.

Bates, D. W., Leape, L. L., Cullen, D. J., Laird, N., Petersen, L. A., Teich, J. M., ... Seger, D. L. (1998). Effect Of computerized physician ORDER ENTRY AND A TEAM INTERVENTION ON PREVENTION OF SERIOUS MEDICATION ERRORS. JAMA: THE JOURNAL OF THE AMERICAN MEDICAL ASSOCIATION, 280(15), 1311-1316.

Benkirane, R. R., Abouqal, R., R-Abouqal, R., haimeur, C. C., S Ech Cherif El Kettani, S. S., Azzouzi, A. A., ... Soulaymani, R. R. (2009). INCIDENCE OF ADVERSE DRUG EVENTS AND MEDICATION ERRORS IN INTENSIVE CARE UNITS: A PROSPECTIVE MULTICENTER STUDY. JOURNAL OF PATient SAFETY, 5(1), 16-22.

Bulhões, I. (2001). Os Anjos também Erram: Mecanismo e Prevenção da Falha humana no trabalho hospitalar. Rio de Janeiro: ATHENEU.

Campanella, D., fraguglia, C., Ferelli, E., Rossi, M., \& Cericola, A. (2013). L'automazione contro gli errori terapeutici. Tecnica OSPEDALIERA, 42-45.

Carvalho, V. T. de, \& Cassiani, S. H. D. B. (2000). ERros na medicaçÄO: anÁlise das SituaçÖEs Relatadas pelos profissionais de enfermagem. Medicina (Ribeiräo Preto), 33(3), 322-30.

Cassiani, S. H. D. B., Teixeira, T. C. A., Opitz, S. P., \& Linhares, J. C. (2005). Medication systems in hospitals and their evaluation by PROFESSIONAL GROUPS. REVISTA DA ESCOLA DE ENFERMAGEM DA USP,

Cohen, M. R. (2006). Medication ERrors (2ND edition). WAShington, DC: AMERICAN Pharmacists Association.

Coimbra, J. A. H. (2008). Prevenção e deteCÇÃo de erros de medicação. ClênCIA, Cuidado e Saúde, 5(0), $142-148$.

COUTO, C. (2013). DeVOluÇÃo de dOSES UNITÁRIAS: ANÁlISE dE UMA PRÁTICA diÁRIA (TítULO DE ESPECIALISTA EM FARMÁCIA). INSTITUTO POLITÉCNICO DO PORTO. RETRIEVED FROM HTTP://RECIPP.IPP.PT/HANDLE/10400.22/3313

Crujeira, R., furtado, C., Feio, J., falcão, F., Carinha, P. H., Machado, F., ... lopes, J. M. (2007). Programa do Medicamento hospitalar. Gabinete do Secretário de Estado da Saúde, Ministério da Saúde.

Dalmolin, G. R. dos S., Rotta, E. T., \& Goldim, J. R. (2013). Medication errors: Classification of SeRiousness, type, and of MEDICATIONS INVOLVED IN THE REPORTS FROM A UNIVERSITY TEACHING HOSPITAL. BRAZILIAN JOURNAL Of PhARMACEUTICAL SCIENCES, 49(4), 793802.

DAWSON, D., \& Reid, K. (1997). FAtigue, ALCOHOl AND PERFORMANCE IMPAiRMENT. NATURE, 388(6639), $235-235$. 
Diz, E., \& Gomes, M. J. (2008). Causas de Erros na Medicação. Revista InVestigação Em Enfermagem, 5-14.

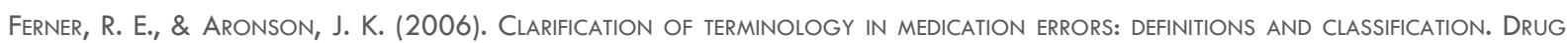
SAFETY, 29(11), 1011-1022.

Ferreira, S., Correia, F., \& SANtos, A. (2012). Interações entre FÁrmacos e Nutrição Entérica: revisão do conhecimento para O DESENVOlVIMENTO DE ESTRATÉGIAS de MINIMIZAÇÃo do RISCO. ARQUIVOS DE MEDICINA, 26(4), 154-163.

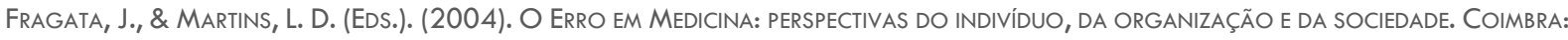
ALMEDINA.

Herdeiro, M. T., Ferreira, M., Ribeiro-Vaz, l., Polónia, J. J., \& Costa-Pereira, A. (2012). O Sistema Português de FarmacovigilânCia. ACtA MÉdica Portuguesa, 25(4), 241-249.

Jesus, Â. (2014). ConceÇão, ImplementaÇão e AValiação de uma Metodologia Blended-Learning, no Ensino da Farmacoterapia baseada em Casos Clínicos (Doutoramento). Universidade Minho, Braga.

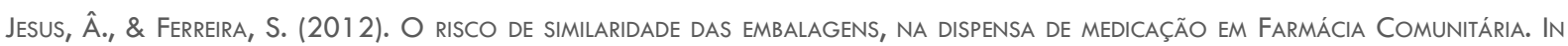
Abstract Book of the 1 St international Health Congress of IPLeiria. Leiria, Portugal.

Kohn, L., Corrigan, J., \& Donaldson, M. (2000). To Err Is Human: Building a Safer Health System. (Committee on Quality Of Health Care in AMerica, InStitute of Medicine, Ed.). The National ACAdemies

Leape, L. L., Bates, D. W., Cullen, D. J., Cooper, J., Demonaco, H. J., Gallivan, T., ... Laffel, G. (1995). Systems analysis of adverse drug eVents. ADE PreVention Study Group. JAMA: The Journal of the American Medical Association, 274(1), 35-43.

López, M. J. O., Merino, M. . V., \& Alvaréz, P. J. M. (2002). PreVención de errores de medicación. Farmacia Hospitalaria, 26(6).

López, M. J. O., \& MUÑOZ, R. M. (2002). PreVención de errores de medicaCión. FarmaCia Hospitalaria, 26(2).

Miranda, P., Pinto, M., Pereira, R., Ribeiro, E., Ferreira, S., Carvalho, A., \& Carinha, P. H. (2012). Reposição do Sistema Semiautomático Pyxis@ Num hospital Central Universitário: Avaliação do Número De Unidades Repostas. In A. Cruz, A. Cunha, A. I. Oliveira, Â. Jesus, C. Pinho, M. Santos, ... R. F. Oliveira (Eds.), Livro de Actas do Vill Colóquio de farmácia (pp. 85-100). Vila Nova de Gaia: Área TéCnICO - Científica de FarmácIa - Escola Superior de TeCnOlogia da Saúde do Porto.

NADZAM, D. M., \& LOEB, J. M. (1998). MEASURING AND IMPROVING THE PERFORMANCE OF HEALTH CARE PROVIDERS: ACCREDIATION IN THE 21 ST CENTURY. AMERICAN JOURNAL OF INFECTION CONTROL, 26(2), 126-135.

National Coordinating Council for Medication Error Reporting and, \& Prevention. (1998). The ncc merp taxonomy of MEDICATION ERRORS.

Otero, M. J. L., JanÉ, C. C., Alonso, M. J. T., \& EnCinAS, M. P. (2003). ERrores de MediCACIÓN: eStandarizaCión de la terminología Y Clasificación Resultados de la BeCA RuIZ-Jarabo 2000. FarmaCia Hospitalaria, 27(3).

Otero, M. J. L., Rodríguez, B. C., Encinas, M. P., Jané, C. C., Alonso, M. J. T., \& Muñoz, T. S. (2008). Actualización de la CLASIFICACIÓN DE ERRORES DE MEDICACIÓN DEL GRUPORUIZ-JARABO 2000. FARMACIA HOSPITALARIA, 32(1), 38-52.

ReAson, J. (2000). HUMAN ERROR: MOdelS AND MANAGEMENT. BMJ : BRITISH MEDICAL JOURNAL, 320(7237), 768-770.

Runciman, W. B., Roughead, E. E., Semple, S. J., \& Adams, R. J. (2003). Adverse drug eVents and medication errors in Australia. International Journal for Quality in Health CARE, 15(SUPPL 1), 149-I59.

TObalQy, M., \& SteWART, D. (2013). EXPLORING HeALTH PROFESSIONALS' EXPERIENCES OF MEDICATION ERRORS IN SAUDI ARABIA. INTERNATIONAL Journal of Clinical Pharmacy, 35(4), 542-545.

VAsconcelos, M., \& Jesus, Â. (2014). ERros de medicação no ato da dispensa - uma perspeCtiva do Profissional de Farmácia. IN atas do X Colóquio de farmácia. Vila Nova de Gaia. 
Wang, J. K., Herzog, N. S., Kaushal, R., Park, C., Mochizuki, C., \& Weingarten, S. R. (2007). Prevention of pediatric medication ERRORS BY HOSPITAL PHARMACISTS AND THE POTENTIAL BENEFIT OF COMPUTERIZED PHYSICIAN ORDER ENTRY. PEDIATRICS, $119(1$ ), E77-85.

WOMER, R. B., TRACY, E., SOO-HOO, W., BICKERT, B., DiTARANTO, S., \& BARNSTEINER, J. H. (2002). MULtidisCiplinaRY SYSTEMS APPROACH TO CHEMOTHERAPY SAFETY: REBUILDING PROCESSES AND HOLDING the GAINS. JOURNAL OF CLINICAL ONCOLOGY: OfFICIAL JOURNAL OF THE AMERICAN SOCIETY OF CLINICAL ONCOLOGY, 20(24), 4705-4712. 